\title{
Multivocal Mapping Studies: Understanding and Application for Interdisciplinary Qualitative Researchers
}

\author{
다성적 주제범위 맵핑 연구: \\ 학제간 질적 연구자를 위한 이해와 활용 \\ Dong-hyun Lee ${ }^{1}$, Soon-jeong $\mathrm{Cho}^{2}$ \\ 이동현 ${ }^{1}$, 조순정 ${ }^{2}$ \\ ${ }^{1}$ Adjunct Fellow, Faculty of Design \& Creative Technologies, Auckland University of Technology, \\ New Zealand,don.lee@aut.ac.nz \\ ${ }^{2}$ Associate Professor, College of Global Regions, Sangmyung University,
}

South Korea,sj.cho@smu.ac.kr

Corresponding author: Soon-jeong Cho

\begin{abstract}
Leading international journals have recently published the literature reviews that belong to multivocal mapping studies (MMS). In response to post-modern critiques, the MMS technique is useful for qualitative literature reviewers who attempt to synthesize published 'white' sources and nonpublished 'gray' literature. It classifies interdisciplinary knowledge in a 'mapping' method by retrieving broad evidence in an applied setting. However, across the Korean academic papers for the practitioner-oriented review, mapping studies in the form of 'multivocality' using white literature and gray literature are hardly seen. This study aims to understand MMS and explain how to apply the method to the arena of literature reviews. To this end, MMS-related information was collected from 18 international and domestic articles published in 1991-2021 to answer: why MMS has emerged?; what the technique is?; how the new approach is applied to literature studies?; which skills differ from other literature research in searching resources and representing knowledge?; and on what the underlying guidelines are based? Thereby what advantages and disadvantages the reviewers, learners, and journal readers in the disciplinary field may earn from the outcomes of MMS? This paper sets out for qualitative scholars in practical disciplines and the industry-academic areas where they can apply the MMS procedure for their literature studies. As such, this work may be helpful for not only the interdisciplinary professionals utilizing multivocal literature but also the practice-based researchers seeking an alternative review approach to reveal complex and unknown issues, such as COVID-19's impacts on the real-world situation.
\end{abstract}

Keywords: Multivocal Mapping Study, Qualitative Literature Review, White Literature, Gray Literature

요약: 최근 들어, 해외 저명 학술지에 새로운 방식의 문헌 고찰인 다성적(多聲的) 주제 범위 맵핑 연구(MMS)가 눈에 띤다. MMS 방식은 후기 현대 조류에 부응하여, 출간된 백색 문헌과 미간행된 회색 정보를 합성 후 방대한 학제간 지식과 근거를 시각적인 맵핑을 통해 분류하는 질적 고찰이다. 하지만 국내 학계에서 백색 문헌과 회색 문헌을 고찰하는 학제간 연구는 거의 보이지 않는다. 본 논문의 목적은 $\mathrm{MMS}$ 방식의 문헌 고찰은 왜 출현했고, 어떠한 고찰법이며,

Received: June 20, 2021; ${ }^{\text {st }}$ Review Result: August 05, 2021; $2^{\text {nd }}$ Review Result: September 21, 2021 Accepted: October 31, 2021 
어떻게 수행을 하고 어떤 기법이 필요하며, 수행 지침은 무엇인지 해설함으로써 포스트모던을 추구하는 문헌 연구자에게 $\mathrm{MMS}$ 방식의 이해와 활용을 돕는 데 있다. 아울러, 동 기법을 적용하는 논문이 연구자 및 구독자에게 주는 장단점과 고려 사항도 논하였다. 본 논문은 다성적 문헌에 관심 있는 학제간 연구 전문가는 물론 코로나 19 와 같이 복잡한 미지의 현상을 폭넓게 밝히려는 대안형 문헌 연구자에게 도움이 될 것이다.

핵심어: 다성적 주제범위 맵핑 연구, 질적 문헌 고찰, 백색 문헌, 회색 문헌

\section{1. 서론 및 배경}

21세기 들어, 전통의 방식을 비판하고 기존의 경계를 허무는 포스트모던(Post-modern, 후기 현대)의 조류가 더욱더 거세다[1]. 이러한 개방의 기조 속에 일부 학계는 "유일하게 타당한 표현 방법은 존재하지 않으며 연구자의 다성적(Multivocal)이라는 주제를 중심으로” 분야를 초월하여 새로운 연구 방법을 활용하자는 후기 현대 사상을 역설한다[2]. 이러한 사상은 문헌 고찰에도 적용되었다. 예로, 1990년대 초 학술 데이터베이스의 전자화로 문헌 탐색이 용이해지자, 국내외 학자들은 투명하면서 객관적인 절차로 선행 연구를 조사하는 체계적 고찰(Systematic Literature Review, SLR)을 널리 활용해 왔다[3]. 그에 더해, 학제간 지식의 영역과 개념, 근거의 유형을 질적으로 합성하는 고찰 방식인 주제범위 연구(Scoping Study, SS)도 고안됐다[4]. 많은 저자에게 친숙한 문헌 연구인 SLR, SS 방식은 등재지가 게재한 '백색' 문헌(White literature), 즉 정식 문헌(Formal literature)이 원칙적인 검토 대상이다. 따라서, 학위청구 논문이나 학술대회 발표는 물론 비영리 단체, 공공 기관, 기업체나 전문가가 공개하는 소위 '회색' 문헌(Gray literature)은 학술 고찰의 범주에서 배제되어 왔다. 왜냐면, 회색 문헌은 지식의 적절성과 정보의 신뢰성을 점검하는 엄격한 체계가 뒷받침돼야 했기 때문이다. 하지만, 포스트모던의 개방성에 맞춰 '다양한 목소리' 담도록 다성성(Multivocality)을 주문하는 산학계를 중심으로 학술적 관점의 여과를 거친 회색 문헌 역시 고찰 연구의 대상으로 삼는 다성적 방식이 시도된다.

이에, 최근 융복합 분야 해외 고찰에서는 SS 문헌 방식에 기초하여 백색 문헌과 회색 문헌을 합성 후 지식 정보를 도식화(Mapping)하는 다성적 주제범위 맵핑 연구(Multivocal Mapping Study, MMS)가 늘고 있는 추세이다[5][6]. 그러므로, 국내의 학제간 연구자에게 대안형 질적 문헌고찰 방식인 $\mathrm{MMS}$ 를 온전히 조명해 줄 필요가 있다. 이는 유사한 고찰 기법의 반복보다 다양한 장르에 대한 문헌 검토와 전달 방식이 실용주의 학술 연구의 건강성을 높이기 때문이다[7][8]. 이러한 배경으로 2018년 이후, 해외 학술지에서 MMS에 의한 분야별 문헌 조사가 눈에 띄고 있음에도 국내에 게재된 문헌 연구 가운데 동 고찰법을 쓴 바는 거의 보고되지 않았다. $\mathrm{MMS}$ 는 백색 문헌과 회색 문헌에서 얻은 정보를 합성한 다음, 시각화 기법을 활용하여 재해석하는 질적 고찰로써 신흥 현상을 영역별로 넓게 검토해 보려는 학자는 물론 광범위한 지식을 빠르게 인지해야 하는 일반 독자에게도 유용하여 동 고찰 방법의 활용이 절실하다[5]. 더욱이, 이 방식은 산학 현장을 통한 경험 지식이 계속 축적됨에 따라 현장형 연구의 주제 발굴과 방대한 유관 증거의 맵핑이 효과적인 사회과학 계통 연구에도 확장성이 있다[6].

본 논문은 등재지 간행물 및 미간행 연구물의 주제범위에 맞춘 합성과 맵핑으로 문헌을 고찰하는 $\mathrm{MMS}$ 를 소개하여 문헌 연구자의 이해와 활용을 돕는 데 목적이 있다. 
동 연구는 $\mathrm{MMS}$ 개념을 처음 접할 독자 입장에서 다음과 같이 질문한다. (i) $\mathrm{MMS}$ 는 어떠한 배경에서 출현했고 왜 필요하며, 어떤 특징이 있으며 기존의 문헌 고찰과 무엇이 다른가? (ii) MMS는 어떻게 문헌을 전개하며 어떠한 고려가 필요하고 무슨 장단점이 있는가? 본 1장의 서론.배경에 이어 2장에서 연구 방법을, 본론의 3장과 4장은 $\mathrm{MMS}$ 방식의 이해와 활용에 관해 살피고, 5 장에서 결론.제언을 논한다. 본 논문은 최근 영미권 컴퓨터.정보공학 관점의 $\mathrm{MMS}$ 지침, 회색 문헌의 학술적 활용과 SS 방식에 관한 국내 간호학, 교육학 문헌을 인용하면서 응용 및 융복합 분야의 연구 고찰에 익숙하지 않을 인문·사회과학 학습자도 읽기 편하게 꾸몄다. 본 연구는 코로나19같이 갑작스레 발견된 주변 현상에 대하여, 학술지 논문부터 비출간 문헌까지 증거의 검토가 필요한 학제간 분야의 연구원, 시각적 기법을 동원한 $\mathrm{MMS}$ 고찰이 효과적일 산학 계통의 실무자, 대안적 고찰을 통해 광범위한 지식의 습득과 이론 배경이 필요한 학위 과정생에게 유용할 것이다.

\section{2. 연구 방법}

본 연구는 MMS 방식의 체계적인 설명을 위해, 2020년 12월부터 2021년 4월까지 5 단계에 걸쳐 통상의 SLR 과정에 따라 국내외 유관 선행 연구를 분석하였다[9]. [그림 1]과 같이, 먼저 SLR 1단계에서는 서론에서 제기한 연구 질문에 충실히 답변하도록 세부적인 검색 전략을 마련했다. 이에, 다성적 고찰과 맵핑 방식에 대한 소개는 해외 연구에서 주로 발견되므로 다수의 영미권 문헌을 참고하였고, $\mathrm{SS}$ 개념은 국내 문헌도 함께 조사하였다. 동 연구의 검색어는 영문 연구물은 'multivocal review', 'scoping study', 'mapping study', 'gray(미국식)/grey(영국식)' 등 단.복수 형태로, 국내 문헌의 경우 ‘다성적', ‘주제범위 연구', ‘맵핑 연구' 등 이와 유사한 의미를 지닌 한.영 검색어를 조합하여 총 12 개의 영문과 한글 검색어를 정했다.

\begin{tabular}{|c|c|c|c|c|c|c|c|c|}
\hline Step 1 & & Step 2 & & Step 3 & & Step 4 & & Step 5 \\
\hline $\begin{array}{c}\text { Protocol } \\
\text { (SLR procedure) }\end{array}$ & $D$ & $\begin{array}{c}\text { Search } \\
\text { (SCOPUS/KCI) }\end{array}$ & $D$ & $\begin{array}{c}\text { Selection } \\
\text { (Zotero Lib file) }\end{array}$ & $D$ & $\begin{array}{c}\text { Evidence } \\
\text { (18 literature) }\end{array}$ & $D$ & $\begin{array}{c}\text { Presentation } \\
\text { (Multivocal + MS) }\end{array}$ \\
\hline
\end{tabular}

[그림 1] 본 연구가 적용한 체계적 고찰 5단계 과정 (출처: [9], 저자 도해)

[Fig. 1] Systematic Review in 5-Step (Source: [9], Reformatted by the authors)

또한, 문헌의 검색 기간은 다성적 개념이 국내외에 언급되기 시작한 1991년부터 2021년으로 설정하였고, 최대한 풍부한 정보를 얻고자 학문 분야에는 제한을 두지 않았다. 이어, $2 / 3$ 단계의 문헌 검색과 선별은 구글 스콜라(Google Scholar)를 주된 탐색원으로 쓰되, 해외 문헌은 SCOPUS 등급 학술지와 국내 문헌은 한국학술지인용색인 $(\mathrm{KCI})$ 등재지로 한정했고, 비공개 $\mathrm{KCI}$ 문헌은 학술지 홈페이지, 문헌 유료 제공 사이트에서 전문을 얻었다. 각 문헌의 자원은 공개용 클라우드 서지관리 소프트웨어인 Zotero 버전 5에서 목록을 만든 다음, Zotero 검색 기능으로 같은 저자의 내용이 중복되는 문헌과 정보가 부실해 보이는 문헌은 서지 파일에서 배제했다. 아울러, 4 단계와 5 단계에 걸쳐 선별된 해외 문헌 11 편과 국내 문헌 7편을 통해 MMS방식의 이해, $\mathrm{SS}$ 연구와 맵핑 기법의 특징과 지침 등을 서술하고 맵핑의 개념을 돕는 그림도 부연하였다. 끝으로, 일부의 원문 용어는 연구 맥락이 유사한 여러 편의 국내 문헌을 
참고 후 해석하였다(예. Multivocal: 다성적, Mapping: 맵핑)[2][5].

\section{3. 다성적 주제범위 맵핑 연구의 이해}

\section{1 다성적 고찰이란?}

\subsection{1 배경과 필요성}

문헌고찰 연구는 선행 연구의 학술 증거를 색인하고, 연구 결과를 비평하며 지식의 공백을 인식하는 데 도움을 준다[6]. 그간의 문헌 연구는 다양한 고찰 방식으로 발전했는데, 대안적인 고찰 발굴에 앞선 분야는 의학계이다. 의학 분야는 생명을 다루는 특성상 새로운 발견에 빈틈없는 실험을 해야 하므로, 일련의 작업 중 수집 증거를 체계를 갖춰 합성하는 데 익숙하다[8]. 따라서 연구자가 실험을 마치면 전문가 집단이 발견 사항을 조사하게 되는데, 검증과 평가는 “반복 가능하되(replicable), 투명하며 (transparent), 객관적이고(objective), 편견 없이(unbiased), 엄밀함이 (rigorous)" 생명이다[9]. 이같이 단단한 연구 방법을 지향하는 의학계는 1990년대 초 학술용 전자 도서관의 발달에 힘입어 코크란(Cochrane) 연합에서 소개한 체계적 고찰인 SLR이 개별 연구자의 주관적 검토를 대체한다[10]. 이후, 객관적인 체계성이 널리 인식된 SLR 방식은 의료와 밀접한 보건 간호.정신 분야 외에 근거 탐색에 민감한 문헌정보, 정보교육, 소프트웨어 분과를 거쳐 사회과학 문헌 연구에도 도입된다[11-13]. 한편, 최근 포스트모던에 입각한 학제간 연구에선 지식적 가치가 담겨 있을 다층적인 자료를 활용, 현장 지식의 시사점을 포함한 학문적 함의를 강조한다[14]. 이에, 의학계는 SLR 방식을 토대로 회색 문헌의 정보까지 연구 목록에 담는 다성적 고찰을 시도한다. 그 이유는 자신의 본업에 바쁜 전문의와 비영어권 의학자 가운데 현장의 연구 결과를 소속 기관의 자료실, 심지어 개인 블로그 등 이른바 '회색' 지대에 먼저 공개하는 일이 잦기 때문이다. 회색 문헌은 학술지에 출간된 백색 문헌에 비해 정보의 신뢰성 측면에 논쟁이 있지만 “최신성과 속보성에서 가치를" 인정받는다[15]. 이에 따라, 문헌 고찰 연구에서 회색 문헌의 활용은 정보, 공학, 보건 등 일선 현장과 밀접한 계열에서 선택되는 가운데, 믿을 만한 회색 문헌을 여과할 지침을 통해 백색 문헌과 합성하는 다성적 고찰법이 갖춰진다.

\subsection{2 회색 문헌의 영역 및 검중}

전통적으로, 정식 출간물인 백색 문헌에 의존한 SLR, SS 방식의 문헌 연구가 비출간 회색 문헌도 함께 학문 영역에 끌어와 증거를 논하기 위해선 회색 문헌에 대한 출처 분류가 선결돼야 했다. 1999년 10월 미국 워싱턴에서 열린 4차 Gray Literature 학술 회의는 대학, 정부, 기업, 전문가 등이 만든 회색 문헌의 출처를 [그림 2]와 같은 네 구역으로 나눴다[16][17].

제 1 영역은 출간된 백색 문헌과는 학술 가치가 다른 학위 논문과 학술대회 발표(Proceedings)를 품는 구역이다. 또한, 제 2 영역에는 기관의 공개 문건, 정부의 백서 및 기업체 보고서, 제3영역엔 집단 지성(예. 위키 백과)과 같은 온라인 공개 정보가 존재한다. 끝으로, 제4영역은 인터넷 카페, 블로그, 사회관계망에 인용된 전문가의 연구 문서를 아우른다. 이같이 회색 문헌은 제 1 영역을 넘어 제 2 영역에서 제 4 영역으로 갈수록 문헌의 규모가 커지고 자료의 원천은 다양해짐으로 출처의 파악이 쉽지 않고, 상대적으로 불확실한 학술 견해가 포함될 개연성이 커진다[18][19]. 이에, 특별히 
제3영역과 제4영역 회색 문헌은 AACODS(Authority(권위)-Accuracy(정확성)-Coverage (범위)-Objectivity(객관성)-Date(시기)-Significance(의의))라는 점검표를 활용, 문헌의 검증을 꼭 밟아야 한다([표 1])[17]. 한편, 기존의 문헌 연구의 경우 석.박사 학위 논문이나 학술 대회의 간행물을 백색 문헌으로 품는 관행이나, 이러한 연구는 학술 단체의 심사 규정에 따라 학술지를 통해 게재되지 않으므로 1999년 워싱턴 Gray 문헌 회의는 물론 다수의 국내외 문헌 연구 전문가들 역시 제 1 영역의 결과물 또한 회색 문헌으로 구분하였다[20].

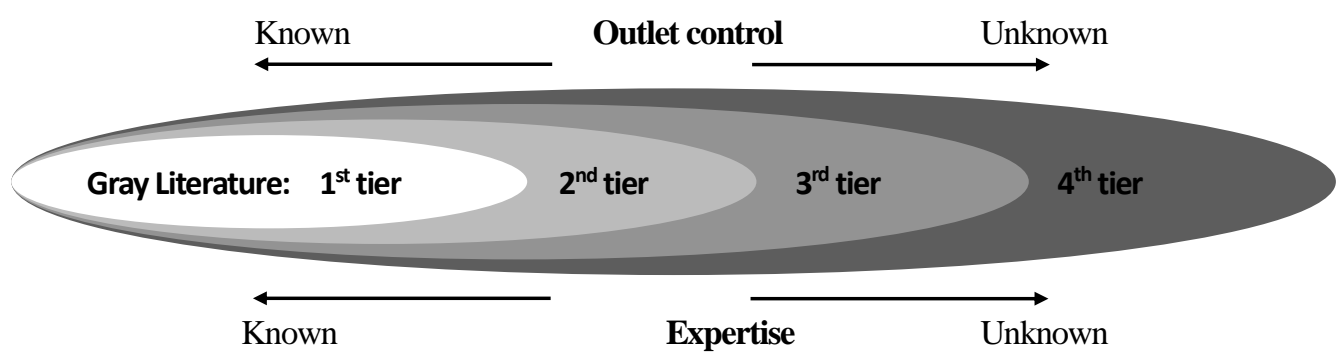

[그림 2] 회색 문헌의 4대 영역 정의 (출처: [16][17], 저자 도해)

[Fig. 2] Definition of the Gray Literature Scope (Source: [16][17], Reformatted by the authors)

[표 1]AACODS 회색 문헌 점검 규약 (출처: [17], 저자 편집)

[Table 1] AACODS for Checking Gray Literature (Source: [17], Edited by the authors)

\begin{tabular}{|c|c|c|c|}
\hline Criteria & Check points & Yes & No \\
\hline Authority & $\begin{array}{l}\text { * Individual authors } \\
\text { - Associated with a reputable organization? } \\
\text { - Produced/Published other work in the field? } \\
\text { - Recognized expert, identified in other sources? } \\
\text { - Cited by others? (e.g., in Google Scholar) } \\
\text { - Higher degree student under expert supervision? } \\
\text { * Common \& Group authors } \\
\text { - Is the organization reputable? (e.g., W.H.O) } \\
\text { - Is the organization an authority in the field? }\end{array}$ & $\left.\begin{array}{ll}( & ) \\
( & ) \\
( & ) \\
( & ) \\
( & ) \\
( & \\
( & )\end{array}\right)$ & 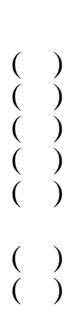 \\
\hline Accuracy & $\begin{array}{l}\text { - Does the item have a clearly stated aim or brief? } \\
\text { - Does it have a stated methodology? } \\
\text { - Has it been peer-reviewed? } \\
\text { - Has it been edited by a reputable authority? } \\
\text { - Is it representative of work in the field? } \\
\text { - Is it an accurate interpretation/analysis? }\end{array}$ & 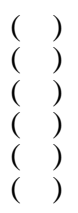 & $\begin{array}{ll}( & \\
( & \\
( & \\
( & \\
( & \\
( & \end{array}$ \\
\hline Coverage & $\begin{array}{l}\text { - Are any limits clearly stated? (e.g., research questions, } \\
\text { parameters, populations, publications, survey, statistics) }\end{array}$ & $(\quad)$ & ( ) \\
\hline Objectivity & $\begin{array}{l}\text { - Is it biased, unstated or unacknowledged? } \\
\text { - Is the author's standpoint clear? } \\
\text { - Does the work seem to be balanced in presentation? }\end{array}$ & $\begin{array}{ll}( & \\
( & \\
( & \end{array}$ & $\begin{array}{l}\text { ( ) } \\
(\text { ) } \\
(\text { ) }\end{array}$ \\
\hline Date & $\begin{array}{l}\text { - Is the easily discernible date a strong concern? } \\
\text { - Can the specified date be closely ascertained? } \\
\text { - Have key contemporary materials been included? }\end{array}$ & $\begin{array}{ll}( & \\
( & \\
( & \end{array}$ & $\begin{array}{l}\text { ( ) } \\
(\text { ) } \\
(\text { ) }\end{array}$ \\
\hline Significance & $\begin{array}{l}\text { - Is the item meaningful and relevant? } \\
\text { - Does it enrich/add something unique to the research? } \\
\text { - Does it strengthen or refute a current position? } \\
\text { - Is it integral, representative, typical and impactful? }\end{array}$ & $\begin{array}{l}\left(\begin{array}{l} \\
(\end{array}\right) \\
(\quad) \\
(\quad)\end{array}$ & 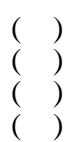 \\
\hline
\end{tabular}


[표 1]와 같이, AACODS 규약은 6대 항목별로 회색 문헌의 발견 근거를 세심히 평가(Critical appraisal)하고, 문헌의 품질과 정보의 신뢰 검사에 적합하도록 구성되어 있어서 비간행 연구 내 증거의 적절성 심사에 유용하다. 특히, AACODS는 인문사회 과학 분야를 포함한 학계 전반에 범용성이 있어서 회색 문헌에도 백색 문헌에 부합하는 권위를 세울 수 있게 한다. 그러므로, 다성적 문헌을 지향하는 연구자는 AACODS 규약을 통해 회색 문헌의 합당한 조사를 마치고 선택된 회색 문헌과 기존의 백색 문헌을 합성할 수 있다. 만약, 동 회색 문헌 검증법이 자신의 연구 조건에 부합되지 않다고 판단되면 연구자는 이유와 대안을 온당히 설명해야 한다.

\subsection{3 회색 문헌의 검색 방법}

회색 문헌은 학술 등재지가 준수해야 하는 권위와 형식에서 탈피하여 최신의 지식을 빠르게 배포될 장점을 지닌 반면, 학술 가치와 정보의 신뢰 면에서 의구심이 적지 않음으로 평소 믿을 만한 연구물을 공개하는 기관이나 저명한 연구자가 공유한 전자 출간에 집중하여 학문 영역에서 다룰 필요가 있다[20]. 따라서, 회색 문헌을 포괄하는 연구는 정식으로 출간된 백색 문헌과 속성이 다른 만큼, 회색 문헌에 대한 접근 전략 또한 다르게 된다[19]. 한편, 회색 문헌에서 어떤 연구물을 포함시키는 점은 연구 주제와 목적에 따른 과업 수행자의 판단이지만, 자칫 고찰 연구의 품질과 가치를 훼손하므로 문헌의 탐색, 선정과 분류에 신중해야 한다. 학계마다 최신 지식과 정보를 담은 가치 있는 회색 문헌의 분량은 미지수이나 대체로 회색 문헌이 차지하는 비율이 클 개연성이 높은데, 이공계 분야의 경우 상호 간 기술 정보의 공유에 더욱 적극적이어서 공개된 회색 문헌의 인용이 매우 많은 편이다[15]. 예로, 해외 소프트웨어 자동화 실험 연구의 경우, 백색 문헌 대비 유의미한 회색 문헌의 비율이 최소 2 배에서 최대 6 배에 달하고, 개발 절차 등 일부의 연구 항목은 회색 문헌이 거의 전량을 차지하고 있다[16].

그 결과, 특정 연구에 맞는 각종 문헌의 탐색과 선정에는 합당한 작업을 필요로 한다. 백색 문헌의 탐색이라면, 자체 검색 엔진이 있는 전자 데이터베이스의 자동 탐색이나 연구자가 속한 학술 단체 웹사이트에서 제공하는 탐색창을 쓴다. 이때, 관심 분야의 전문성을 가진 소수의 출처 간 전후를 탐색하면서 대상 문헌을 확장하는 눈덩이 표집을 병행한다[16][19]. 반면, 회색 문헌을 습득하려는 고찰 연구는 학위 논문 웹사이트, 학회의 학술대회 홈페이지, 대학별 전자 도서관과 인터넷 공개 자료원을 상대로 한 수동 검색에 의존해야 한다. 따라서 연구자가 웹 브라우저를 통해 정밀하고 효과적인 추출이 필요할 경우는 논리 연산자를 써서 추출한다[18]. 이 경우, 논리 연산에 쓰이는 기호는 보통의 검색 엔진에서 호환되는 $\mathrm{AND}$ (논리곱), $\mathrm{OR}$ (논리합), $\mathrm{NOT}$ (논리차), 큰따옴표("”) 등이다. 예로,“대학”, “경영”을 동시에 또는 하나만 포함할 검색 연산이면 두 단어 사이에 AND나 $\mathrm{OR}$ 을 넣는다. 이와 같이, 큰따옴표는 특정 어구(예. "외국인 유학생 평가") 검색에 긴요하며, $\mathrm{AND} / \mathrm{OR} / \mathrm{NOT}$ 과 같은 기본연산자, 인접연산자, 와일드카드(*) 등과 조합도 가능하다.

\section{2 주제범위 맵핑 연구의 이해}

오늘날 포스트모던의 연구자는 다층적, 입체적 형태로 지식을 전달하기 위해 “인식의 지도 작성(Cognitive mapping)"에 의한 과학적 지식을 재현한다[14][15]. 주제범위 고찰인 $\mathrm{SS}$ 연구는 SLR과는 전개 방식의 개념에서 다르다. SS 방식은 현존의 지식을 체계 있게 
수집 후 합성하여, 관심 있는 연구의 주요 개념, 문헌의 양상, 근거의 유형을 통해 분류하는 탐색적 고찰이다[12]. 특히, SS 고찰은 SLR 연구물과 달리 연구 질문에 비평적으로 답하거나 세부적인 발견 사실에 초점을 두지 않으며, 각종 개념, 사상과 문헌 근거를 특정 영역과 주제에 맞춰 직관적인 분류에 중점을 둔다[3]. 이러한 SS 방식에 더하여 소프트웨어 공학계는 [그림 3]과 같이 예시한 섬네일과 같이 각 정보의 유형을 도식적으로 분류하는 맵핑 기법을 결합, 특정 지식의 영역을 지리적 형태로 묘사하고 발견된 사실을 구조적 관계로 재해석하는 MS 고찰을 제시한다[21]. 그렇기에, MS 방식은 방대한 문헌 지식의 분류와 발견된 결과의 시각화에 중점을 둔다. 근래에는 컴퓨터를 활용한 디자인 기술의 발달로 인해 전통적인 텍스트와는 다른 이미지(예. 개념 지도)로 연구 결과를 창의적으로 재현하기 용이하다[22].

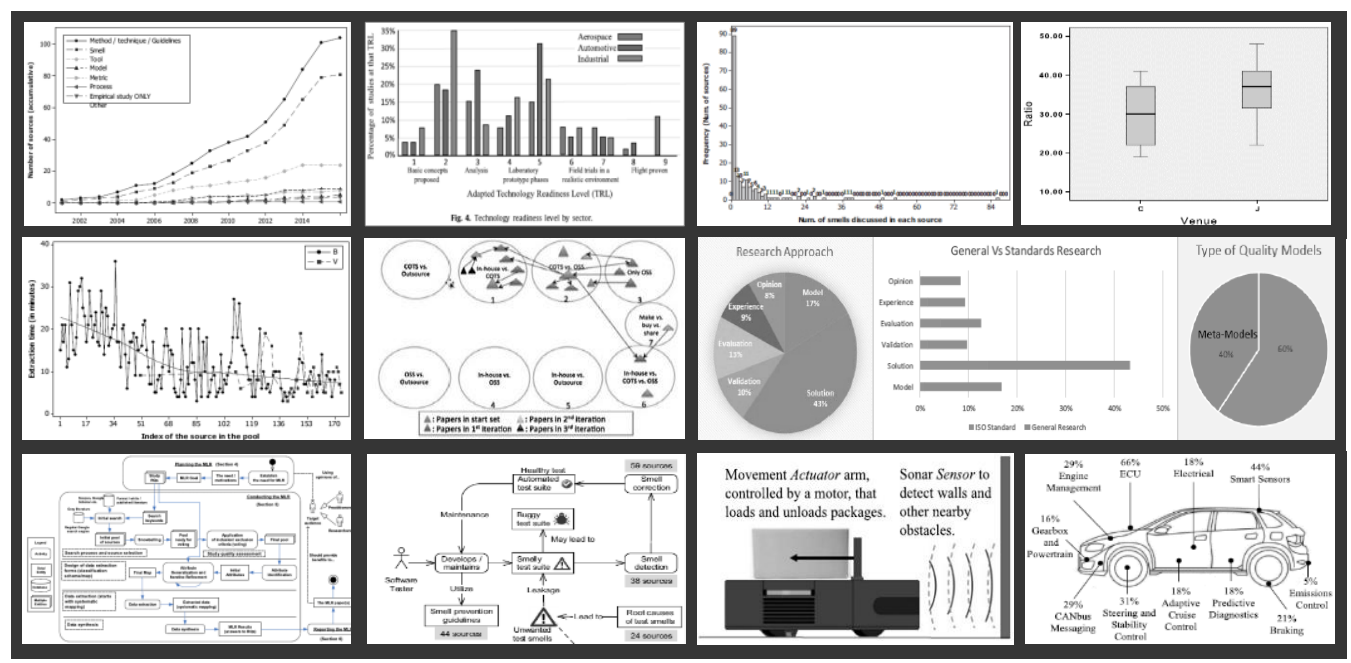

[그림 3] 축약 그림: 주제범위 맵핑 연구물 도해의 예 (출처: [6][16][18][23][24], 저자 도해)

[Fig. 3] Thumbnails: Diagrams in Mapping Studies (Source: [6][16][18][23][24], Edited by the authors)

이에, 종전의 $\mathrm{SS}$ 연구는 문자와 도표(Table/Figure)로 내용과 결과를 서술했다면, $\mathrm{MS}$ 연구는 주로 차트(Line/Bar/Flow/Combo), 분포도(Histogram), 박스 플롯(Box plot), 관계도 (Network), 삽화/화보(Illustration), 2/3차원 다이어그램(2/3D diagram) 등 다양한 도식으로 고찰을 강조한다. $\mathrm{MS}$ 방식이 도식화에 중점을 둔 이유는, 공학.의학 분야의 현장 전문가들이 시각화 도구를 써서 초기 모델링, 가상 테스트, 종합 결과를 공간 효율적으로 재현하는 데 익숙하기도 하지만 단일 연구물에 여러 주제를 소화해야 하는 문헌 연구의 특성상 맵핑 방식이 “초록(Abstract)”을 열거하는 효과를 내기 때문이다[21][25].

\section{3 주요 문헌 고찰 방식의 비교}

앞선 고찰 기법의 이해를 바탕으로 SLR과 SS 방식에 맵핑 기법이 부가된 MS 연구의 주요 특징을 [표 2]로 정리하였다. 먼저, 기존의 SLR 연구는 문헌 내 지식의 분석과 비판을 목적으로 하는 반면, MS 형식은 문헌의 주제와 지식의 조사가 목적이기에 SLR 연구에 앞서 큰 틀에서 문헌 정보를 제공할 때 적합하다. 
[표 2] 체계적 고찰 vs. 주제범위 맵핑 연구 (저자 생산)

[Table 2] Systematic Literature Review versus Scoping Study + Mapping (Produced by the authors)

\begin{tabular}{|c|c|c|}
\hline Systematic Literature Review (SLR) & Focus & Scoping Study (SS) + Mapping (MS) \\
\hline Analysis and criticism of knowledge & Research purpose & Summary of knowledge and evidence \\
\hline Finding knowledge/evidence in particular areas & Role/Function & Providing research information across fields \\
\hline Based PICOC & Question framework & - \\
\hline Specific (e.g., used variables) & Direction/Results & Generic (e.g., research trends) \\
\hline Based on unstructured texts & Presentation & Based on structured images/tables \\
\hline Family oriented & Approach & Species focused \\
\hline
\end{tabular}

이에, SLR방식은 문제 관찰에 깊이를 담고자 PICOC(Population(개체)-Intervention(조정)Comparison(비교)-Outcome(결과)-Context(맥락)) 규약을 써서 연구 질문을 만든다[4]. 하지만 MS 기법 연구는 문헌의 유형 분류에 초점을 두므로 연구 질문이 포괄적이다. 연구 결과의 전개에 있어, SLR 방식은 문헌이 활용한 변수의 나열 등 구체적인 반면, $\mathrm{MS}$ 체계는 기존 연구의 양상을 묘사하므로 보다 개괄적인 편이다. 끝으로, 연구의 결과 전개에서 SLR은 일부 도표를 포함한 문장 중심으로 서술함에 비해, MS 연구는 구조화된 도해 중심으로 고찰 내용을 전한다.

한편, 전통적인 SLR과 SS, MS 고찰은 백색 문헌에 의존해 왔지만 학문적 엄밀보다 실용성에 무게를 두는 일부 학제간 연구를 중심으로 회색 문헌도 고찰 대상에 포함하는 추세임을 앞서 설명했다. 그러한 관계를 [표 3]에서 보면, SLR과 MMS는 백색/회색 문헌 등 자료원의 속성 및 결과의 전개에서 대칭 관계에 있다. 다성적 연구의 핵심은 백색 문헌과 회색 문헌으로 얻은 근거 자료의 합성(Synthesis)이다. 비록, 회색 문헌만을 탐색하는 고찰 연구(예. GLR, GSS, GMS)가 공중 보건 등 현장 분야 외에 전통적인 학계에서는 아직 드문 가운데, 다성적 고찰 연구(예. MLR, MSS, MMS)는 2010년대 후반부터 해외의 실용학자(Pragmatist)를 중심으로 종종 소개된다[26]. 전반적으로, SLR은 고찰의 성격이 지식의 과(Family)를 조사하며, $\mathrm{MMS}$ 는 검토 주제에 대한 종(Species)을 파악하는 데 있다고 간주한다[3]. 따라서, MMS 연구의 수행에는 단계별로 특별한 수행 과정을 요구하고 있으며, 이는 다음 절에서 살핀다.

[표 3] 주요 문헌 고찰의 비교 - 각 문헌 자료원 및 전개 방식 (저자 생산)

[Table 3] Highlighted Literature Studies - Data Sources \& Presentations (Produced by the authors)

\begin{tabular}{|c|c|c|c|c|}
\hline \multirow{2}{*}{ Selected literature } & \multirow{2}{*}{ Data sources } & \multicolumn{2}{|c|}{ Presentation focus/skills } & \multirow{2}{*}{ Major users } \\
\cline { 3 - 5 } & & Scoping & Mapping & Academic researchers \\
\hline White literature & SLR & SS & MS & Industry practitioners \\
\hline Gray literature & GLR & GSS & MMS & Pragmatic researchers \\
\hline Multivocal & MLR & MSS & \multicolumn{2}{|c|}{ Species } \\
\hline Biological similarity & Family & \multicolumn{2}{|c|}{ - } \\
\hline
\end{tabular}




\section{4. 다성적 주제범위 맵핑 연구의 활용}

\section{1 다성적 주제범위 맵핑 연구의 수행}

최근의 질적 연구자는 가치 있는 지식 정보를 탐색하고 재현하는 데 있어, 영역별로 취득한 자료를 보다 효과적으로 분석하며 체계적으로 전개하는 기법을 구사할 필요가 있다[27]. 앞서 보았듯이, MMS는 학계에서 발간한 문헌에 미발간 연구물을 모은 회색 문헌을 더하여 SS 방식에 맵핑 기법을 활용하며 지식의 주제범위를 밝히는 체계를 지녔다. 이러한 특성으로, $\mathrm{MMS}$ 에는 학술 관점의 백색 문헌과 실용 중심의 회색 문헌을 합성 후 방대한 지식과 영역별 근거를 시각적으로 배열하면서 두 고찰 자료원 간에 균형과 조화를 이뤄 설계하는 것이 중요하다[6]. 예로, 실용 중심의 학계에서는 $\mathrm{MMS}$ 작업 시 연구 질문에 $\mathrm{PICOC}$ 규칙을 적용치 않는 대신, 신뢰 있는 회색 문헌의 선별을 위하여 AACODS 규정을 쓰는 점을 앞에서 설명했다[21]. 하지만 문헌 조사를 체계적으로 실행케 함으로써 연구 시각의 편향을 최대한 줄이며, 당대의 해당 분야에 축적된 지식에 뜻있는 보완과 발전을 유도하는 점은 공통적이다[12]. 결론적으로, MMS 연구는 체계적 고찰에 회색 문헌을 포함시킨 다성적 고찰 방식에 도해에 의한 주제범위 방식이 결합된 특성을 지녔다. 그럼에도 회색 문헌의 수용은 출간 문헌으로는 부족한 전문성을 시험적으로 보완하는 정도로 통제를 함으로써 본질적으로 SLR 이나SS 방식이 추구하는 엄밀성, 객관성, 신뢰성을 훼손치 않는 고찰이어야 한다[17]. 따라서, 질적 분석과 맵핑 작업을 수행함에 앞서 합당한 문헌의 탐색과 적절한 증거의 분류가 중요하다[18].

\subsection{1 수행 과정과 지침}

현장 출신의 질적 연구자는 자료를 수집하고 분석하는 방법 외에 영역, 범위와 주제에 따라 추려낸 결과를 어떤 과정과 방식으로 “재현하고 전달할까" 고민해야 한다[27]. 앞에서 본 MMS 방식의 기본 개념을 유념하면서, 실제 MMS 기반의 문헌 연구에 필요한 3단계 과정 및 수행 지침을 [그림 4]와 함께 살펴보고자 한다. MMS 연구의 단계는 회색 문헌에 대한 별도의 접근과 맵핑이 필요한 점 외에는 고찰 과정을 중요시하는 $\mathrm{SS}$ 체계가 유지된다.

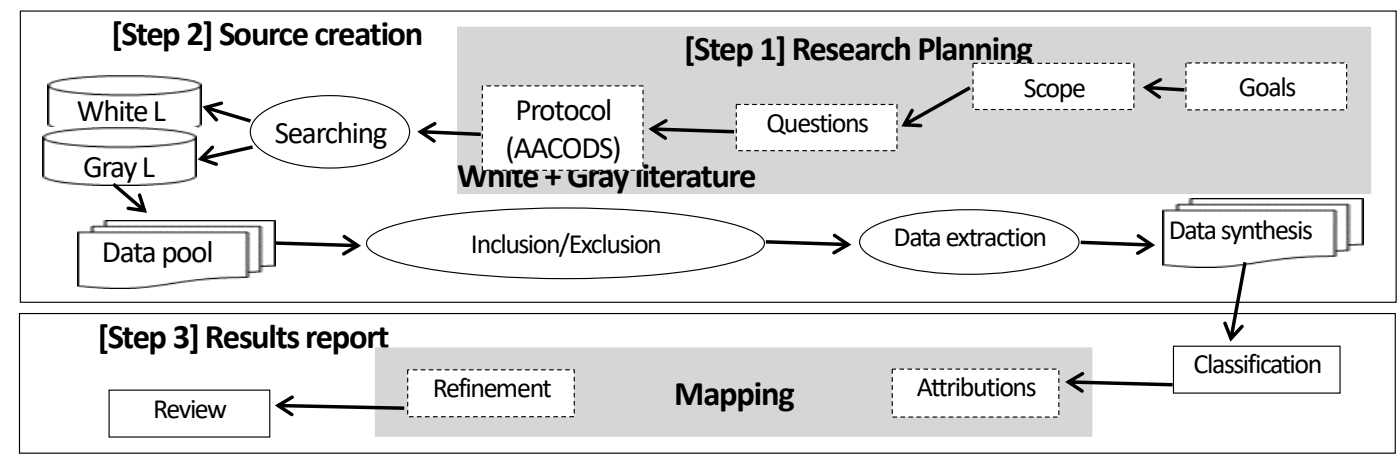

[그림 4] 다성적 주제범위 맵핑 연구의 수행 3단계와 주요 과정 (출처: [6][19], 저자 편집)

[Fig. 4] Research Steps/Procedure for Multivocal Mapping Study (Source: [6][19], Edited by the authors)

[1단계] 계획 수립: 영역 결정 $\rightarrow$ 문헌 파악 $\rightarrow$ 연구 질문 $\rightarrow$ 규약 마련 $\rightarrow$ 규약 검토 [2단계] 자료 생성: 문헌 탐색 $\rightarrow$ 문헌 선정 $\rightarrow$ 품질 확인 $\rightarrow$ 자료 추출 $\rightarrow$ 자료 합성 
[3단계] 결과 보고: 증거 전개 $\rightarrow$ 문건 생산 $\rightarrow$ 연구 평가

1 단계는 연구 계획을 수립하는 5 개의 하위 과정을 포함하며, 우선 문헌의 영역과 주제를 정하고 백색 문헌과 회색 문헌의 접근 방안을 마련한다. 이어, 동 단계의 핵심인 연구 질문을 제시 후, AACODS 등을 기초로 한 문헌의 선정 규약을 확정한다. 2단계는 1 단계에서 목록화 한 출처를 온라인으로 탐색 후 믿을 만한 연구를 택한다. 특히, 기 수립한 규약을 토대로 회색 문헌의 적정성을 파악하고 자료를 추출, 백색 문헌과 자료를 합성을 포함하는 5 개 과정을 행한다. 3 단계는 연구 결과의 보고이며, 2 단계에서 수집한 증거를 주제범위로 맵핑하여 연구물을 생산하고, 해당 분야의 전문가 집단과 연구 결과를 평가한다. 아래는 $\mathrm{MMS}$ 각 단계에서 주요 과정인 (1) 연구 질문, (2) 선정 규약, (3) 탐색 전략, (4) 선정 과정, (5) 자료 추출, (6) 결과 보고의 세부 설명 및 실제 문헌의 <예>이다[3][12][19].

(1) 연구 질문: MMS 연구는 문헌의 영역이 다양하고 주제가 광범위하다. 앞선 설명과 같이, SLR 체계는 PICOC 형식에 맞춰 연구 질문을 구성하나, MMS 연구 질문은 새로운 현상을 회색 문헌까지 광범위하게 검토하므로 $\mathrm{PICOC}$ 체계에 의존치 않고 질의가 포괄적인 편이다[4]. 하지만 질문의 내용은 명확히 서술하여, 문헌 검색 등 후속 전략을 꾸민다. 연구 목적에서 어떤 범위와 측면을 강조할지 고민하여 명료한 질문을 세운다[12].

<예> 국내 연구: 간호 대학생 동료 멘토링에 대해 기존 문헌에서 알려진 것은 무엇인가?

(2) 선정 규약: MMS 연구에서 백색 문헌은 연구 목적에 따라 충실히 선정하되, 해당 분야에 초보인 문헌 연구자는 백색 문헌 역시 여과 기준을 적용한다. 하지만, 회색 문헌에 대한 규약은 AACODS 점검표를 써서 연구 수행자가 직접 구성하고, 해당 점검표는 연구 분야의 권위자, 실무 전문가의 비평과 검증을 받아 품질 있는 회색 문헌의 탐색을 꾀한다[6].

<예> 해외 연구: 테스트 결과의 회색 문헌 공개자는 $\operatorname{IEEE(ㅈㅓㄴㅈㅏㅈㅓㄴㄱㅣㄱㅣㅅㅜㄹㅈㅏㅎㅕㅂㅎㅚ)~ㅎㅚㅇㅝㄴㅇㅔ~}$ 한정

(3) 탐색 전략: MMS의 문헌 탐색 전략은 기존의 SLR 연구처럼 체계적이되, SS 방식과 같이 포괄적이기도 하다. 2000년대 이후 인터넷을 통해 발달한 전 세계 전자 도서관과 유·무료 온라인 학술 데이터베이스에 의존하되, 문헌 제공 웹사이트의 키워드 자동 검색과 연구자의 논리 연상에 의한 수동 검색을 병행한다. 회색 문헌 중 학위 논문과 학술대회 발표는 학술용 검색 엔진인 구글 스콜라에서 목록을 만든 후, 각 대학교와 학회 사이트에서 원본을 얻는다[6]. 회색 문헌은 학계의 권위자나 공신력 있는 기관이 게시한 정책 보고, 연례 백서, 전문가 기고가 권장된다. 다성적 탐색은 문헌 작업에 시간과 인력이 소요되므로 자료의 수집과 분석 반복 후 도출된 내용이 유사해지는 포화(Saturation)에 이르게 되면, 작업을 마감하고 공백 부분은 연구의 한계로 선을 긋는 결단도 필요하다[3][16].

<예> 해외 연구: “미국” AND “장애인교육법” AND 교사의 책무 OR 부모의 권리

(4) 문헌 선정: 문헌의 선정 과정에 있어 생명은 엄격성과 객관성이므로 이전 단계에서 마련한 선정 규약에 집중하되, 심지어 해당 분야에서 저명한 학자의 논문이라도 연구의 목적에 무관해 보이면 과감히 제외한다[9]. 만약, 문헌의 내용은 우수하나 연구 시점, 작성 언어, 학술지 등급 이슈에 따른 포함/배제 여부는 AACODS 점검표에 따르고 연구 동료들과 상의 후 판정한다. 특히 회색 문헌은 넓은 범주를 다루므로, 동 점검표에 
기반하여 재현성, 투명성을 높이고 훗날 제3자 심사에 대비하여 선정 사유도 기록한다[4]. <예> 해외 연구: 1 차 선별된 회색 문헌에 AACODS 항목별로 각자 점수 부여 후 공동 선정

(5) 자료 추출: MMS 연구에서 자료의 추출은 고된 작업이다. 먼저, 문헌의 저자, 연도, 지역, 제목, 키워드, 방법론 등 기본적인 서지 자료에 이어 분류 방식, 시각 기법과 도해의 종류 등도 포함시킨다[18]. 문헌의 자료 기입은 스프레드시트를 써서 하위 주제와 범위를 시트 및 필드 별로 나눈다[28]. 문헌 내 문맥과 정황을 파악해야 할 경우엔 질적 분석을 위한 코딩 작업을 거친다[4]. 이 경우, 주로 개방형 코딩(Open coding) 및 축코딩(Axial coding)에 의한 내용분석을 쓰는데, 추려진 내용이 복잡할 때 백색.회색 문헌을 통한 고찰 결과의 질적인 합성을 위하여 정보를 재해석해야 하기 때문이다. 최종의 분석 자료는 1 인 연구자의 단일 검수에 의존하기 보다는 자료의 수집 중 틈틈이 전문가의 검토를 받고, 추출 작업이 장시간 중단되는 사태에 대비, 향후에 작업을 원활히 재개할 수 있도록 연구 노트를 잘 유지한다[16]. 추출 분량이 많을 경우, 초도 연구와 2차 연구로 나눈 단계적 방식도 고려할 수 있다[19].

<예> 해외 연구: 선정된 회색 문헌을 6 가지 문헌 주제의 3 대 지식 영역에 따라 분류

(6) 결과 보고: 백색 문헌과 회색 문헌을 통하여 각각 취합된 자료는 내용을 합성하여 주제와 범위에 따라 맵핑하여 결과를 보고한다. 이때, 증거 자료를 지나치게 포괄적으로 합성하여 후속의 SLR 연구에서 중요한 의미를 놓치는 일이 없도록 한다[16]. MMS 연구를 보는 계층이 순수 학계의 연구자인지, 실용 분야의 학제 전문가인지 미리 고려하되 그 계통의 독자 중심으로 일관성 있게 결과를 전개한다[3]. 이때, 자신의 분과에서 흔히 쓰는 주제의 구조화 기법이나 실무 직종의 분석적 얼개를 통하여 연구 양상을 묘사하면 효과적이다[12]. MMS 연구물은 광범위한 논제를 다루는 특성이 지니므로, 시각적으로 창의와 개념이 있는 도해 기법을 고안하여 독자의 이해력과 가독성을 돕도록 한다[23].

<예> 국내 연구: 문헌 동향을 논문 목적, 참여자, 학습자 등 6 개 관점에 따라 8 개로 시각화

\subsection{2 연구 수행의 예제}

해외 산학계에서도 아직 소수인 MMS 연구물 중에서, 본 저자들의 식견 상 충실한 작업 과정을 담았다고 판단된 경영정보 학계의 선행 MMS인 Garousi \& Küçük(이하 두 개발자)의 예시를 살펴본다[18]. 두 개발자는 소프트웨어 테스트 과정에서 나온 착오는 아니지만, 점검 관리 체계가 허술하여 훗날 자잘한 문제를 유발할 냄새를 풍기는 소위 'Test smells'라고 칭한 각종 테스트의 '낌새' 유형을 모으고자 고찰 연구를 시도했다[표 4]. 먼저, 1 단계 연구계획 수립에 있어 두 개발자는 관찰할 소프트웨어 분야의 영역을 특정한 다음, 동 연구 문제가 산업계의 소프트웨어 개발 현장이라는 특성상 학계의 문헌보다 현장의 비출간 경로에 갖가지 낌새를 다룬 근거가 산재해 있다고 판단, 회색 문헌을 시키기로 한다. 이에, ‘해당 분야의 테스트에서 어떠한 낌새가 관찰되는가?’를 위시한 8 개의 연구 질문을 제기 후 9 가지 도해 기법에 의한 MMS 고찰법을 채택한다. 
[표 4] 다성적 주제범위 맵핑 연구의 실행 사례 (출처: [6], 저자 편집)

[Table 4] An Exemplar of Multivocal Mapping Study (Source: [6], Edited by the authors)

\begin{tabular}{|l|l|l|l|}
\hline \multicolumn{2}{|c|}{ Step/Procedure } & \multicolumn{1}{|c|}{ Actions } & \multicolumn{1}{c|}{ Applications/Examples } \\
\hline \multirow{5}{*}{ Step 1 } & Scope definition & Software development area & Based on testing scheme on JFreeChart homepage \\
\cline { 2 - 4 } & Initial research & Literature in recent 5 years & On Google Scholar + Software specialist websites \\
\cline { 2 - 4 } & Research questions & What kinds of test smells? & 8 questions and 9 answers \\
\cline { 2 - 4 } & Selection criteria & \multirow{4}{*}{ Criteria for gray literature } & A total of 17 elements on AACODS \\
\cline { 2 - 4 } & Protocol review & AACODS review by 3 specialists (via online voting) \\
\hline \multirow{5}{*}{ Step 2 } & Search strategy & Algorithm/Expression & Google PageRank and Boolean operators \\
\cline { 2 - 4 } & Literature search & Manual by authors & 46 White L. and 120 gray L. based on AACODS \\
\cline { 2 - 4 } & Quality checks & Co-work with experts & Realtime discussions with Google sheet \\
\cline { 2 - 4 } & Data extraction & Meaningful case downloading & Links/Web address on Google sheet \\
\cline { 2 - 4 } & Data synthesis & Open coding + Axial coding & Contexts and themes on 'Test smells' \\
\hline \multirow{5}{*}{ Step 3 } & Evidence mapping & Initial attribution & 8 large attribution and 7 detailed attribution \\
\cline { 2 - 4 } & Results representation & 13 mapping skills & Mapping skills using 13 tables/charts/diagrams \\
\cline { 2 - 4 } & Study review & Peer review in the lab & Modification and revisions \\
\hline
\end{tabular}

아울러, AACODS 점검표에 따라 17 개의 점검 항목을 만들어 회색 문헌을 추려낼 규약을 마련하고 세부 규약에 관한 적합성을 전문가와 확인한다. 두 개발자는 또한 연구 자료를 생성하는 2 단계에서 낌새 정황이 담긴 테스트 문헌의 탐색에 구글이 제공하는 각종 서비스와 자신들이 개발한 연산자를 써서 최근 5 년의 제 $3 / 4$ 영역 회색 문헌 120 편과 학술지의 백색 문헌 46편을 선별한다. 그 과정 중, 먼저 작성한 AACODS 점검표를 통해 분야 전문가와 온라인 검토를 수행하며 회색 문헌의 적절성을 평가한다. 이후, 소프트웨어 개발자 모임 전문 사이트에 접속하여 낌새가 담긴 테스트 자료를 내려 받은 후, 개념별로 테마를 찾는 개방형 코딩과 각 테마를 필드별로 분류하는 축코딩을 실시, 백색/회색 문헌의 정보를 합성한다. 끝으로, 3 단계의 연구 결과의 보고는 9 가지 기법으로 생성된 13 개의 도해를 주제별로 분류 후, 소프트웨어 개발 전문가들과 동 연구 주제인 테스트 낌새의 양상을 검토하면서 각각의 낌새 유형을 확정하는 과정이다.

\section{2 연구의 고려 사항}

\subsection{1 시점과 정황}

그럼, 어떤 시점과 정황에서 $\mathrm{MMS}$ 방법에 의한 고찰이 적합한가? 첫째, MMS 방식은 신규 법제, 신흥 시장, 신종 기술, 신생 서비스로 우리 주변에 환경적, 제도적, 기술적으로 새로운 현상이 발현된 시점이다[6]. 종전에는 학계의 관심을 끄는 이슈에 관한 문헌이 어느 정도 규모를 이루면 SLR 방식의 고찰 연구가 나오곤 했다(예. 2010년대 초 게이미피케이션). 하지만 사회와 시장 저변에 파급력 있는 이례적인 현상은 깊이 있는 문헌 연구에 앞서 큰 틀의 주제와 범위로 배경 정보를 수집, 연구 시각을 제시할 필요가 있다. 이는 2020년 초부터 국내외 각계에서 쏟아지는 코로나19에 관한 연구가 대표적이다. 둘째, 특정 분야의 문헌 분석과 메타분석 연구가 많지 않은 상황이다. MMS 목적은 학술 지식과 실용 지식을 합성하여 문헌의 주제와 범위를 제시하는 데 있으므로, 광범위한 연구 질문의 답변에 유용하고 각 문헌의 구체 증거를 파악하며, 다른 각도의 연구 통찰에 유용한 정보와 향후의 연구 방향을 제시할 때 좋다[3]. 이러한 다성적 
접근에 따른 고찰 증거는 이어서 문헌의 메타 연구를 촉진하는 작용도 한다[16]. 셋째, 특정 현상에 대해 세밀한 모델링 수립이나 모의.가상 실험 준비가 필요한 때이다[21]. 특히, 이공 계열과 학제간 분야 등 실용 지식과 현장 배경이 절실한 분야에 종사함에도 모델링 등 현장 지식에 대한 개념화가 약한 학위 과정자라면, 연구 초기에 권위 있는 전문가가 공유한 미발간 문헌과 도해 중심의 $\mathrm{MMS}$ 에서 연구의 통찰을 얻을 수 있다. 예로, 정보경영 학계의 경우 순수 이론적 연구는 적은 반면 실험 검증과 시뮬레이션에 관한 응용 및 융복합 연구가 $20 \%$ 에 달하므로, 다성적 고찰을 위해 검증된 회색 문헌의 출처를 모은 웹사이트도 운영되고 있다[19][24].

\subsection{2 주요 장단점}

MMS 고찰을 행하거나 연구물을 접하게 될 산학 연구자, 행정 정책가, 산업 현장의 전문가, 고등교육 학습자와 일반 구독자가 느낄 장점은 먼저, 향후의 고찰 연구에 주는 시간과 노력의 절약이다. 이는 선행된 $\mathrm{MMS}$ 결과물을 참고함으로써, 심층적인 SLR 방식의 검토를 이어서 행할 문헌 연구자에게 주는 시간적 이점과 함께 신흥 현상에 관한 연구 동향의 이해나 방향 설정에 도움을 주는 점이다. 또한, 특정 영역의 문제를 처음 접하는 학자에게 직관적인 도해를 바탕으로 문헌 전반의 이해를 제공할 수 있다. 전반적으로, 연구 방법론적 지식 없이 독해가 가능한 점, 도식화된 전개로 신속한 이해를 줄 수 있는 점, 특정 현상의 주제범위 분류에 따른 유관 지식의 공백 인지가 용이하다. 반면에, MMS 고찰의 단점은 독자가 전반적인 문헌의 배경 정보에 의존함으로써 인용된 문헌을 별도로 정독하지 않을 개연성이다. 이에 따라, MMS 연구를 참고한 후속 연구자가 해당 주제의 학술 지식에 오해를 낳을 수 있고, 합성된 문헌의 정보 재현과 지식의 분류가 옳지 않다면 이어지는 세부 연구의 고찰 작업 시 혼선을 빚게 할 수 있다. 아울러, MMS 작업자에게는 광범위한 문헌의 증거와 근거 정보를 도식화해 가며 지식을 담는 작업 과정이 고된 점이다.

\section{5. 결론 및 제언}

지금까지 살핀 바와 같이, 근거 자료의 체계적 요약과 주제별 지식의 합성이라는 목적에 따라 개발된 문헌 연구인 MMS 방식은, 문헌 연구 출간된 백색 문헌과 출간되지 않은 회색 문헌을 함께 고찰하고 해당 분야에 맞는 시각적인 기법으로써 방대한 지식을 효과적으로 전개함을 알 수 있었다. 따라서 학제간 연구가 더욱 활발하고 종전의 경계를 허무는 개방이 역설되는 시대를 맞아, 정식의 출간 문헌과 함께 품질과 가치가 확인된 분야별 회색 문헌도 참고한 학술 연구는 실용 이론의 공백을 메꾸고 현장 지식의 경계를 넓혀줄 수 있다. 이에, 앞으로 학제간 고찰 연구는 그간 익숙한 SLR 방식의 준거 이론의 유형, 개념 모형의 특성, 인과 변수의 정리, 주제어 분석을 넘어 산업 현장의 지식을 담은 정보를 합성하면서 새로운 차원의 고찰 체계를 시도할 필요가 있다. 더욱이, 실무 현장에서는 각종 연구 문제들이 빠르고, 지속해서 보고되고 있는데, 기존의 학술적인 지식과 현장의 방대한 지식을 담은 회색 문헌을 정제, 합성한 질적 연구를 통하여 보다 창의적인 문제 해결법을 모아야 할 시대이다[29][30]. 기존의 문헌학자는 주로 SLR 방식으로 고찰 과정의 체계성, 엄밀성과 명료성을 높이고 개인의 주관적인 고찰 관행을 극복해 왔다[9]. 이에 더해, 회색 문헌이 추가되고 SS 고찰 방식에 맵핑 개념을 결합한 $\mathrm{MMS}$ 방식은 광범위한 지식과 증거를 주제와 영역에 따라 근거를 시각적으로 
구성하므로, 포스트모던을 추구하는 최근의 시대적인 연구 기조에 부합된다.

본 연구는 Multivocal Mapping Studies: Understanding and Application for Interdisciplinary Qualitative Researchers-다성적 주제범위 맵핑 연구: 학제간 질적 연구자를 위한 이해와 활용을 표제로, 국내 학술 분야에서 보기 힘든 $\mathrm{MMS}$ 방법론에 관한 고찰 연구의 길잡이가 되는 총론 성격의 연구라는 시사점이 크다. 비록, 현재 MMS 연구물은 해외에서도 한정되어 있어 다양한 활용 사례를 나열하기 힘들었지만, 문헌 고찰에 관한 새로운 추세를 이해하고 앞으로 국내외 융복합 학계 간 이를 공동으로 활용할 인식을 심어 주었기에 본 연구는 의의가 있다. 더욱이, MMS 방식은 양적인 합성이나 내적 타당성에 대한 연구물의 지적 평가 없이 각종 문헌이 가지는 단면을 보여주고 투명한 기준에 따라 검증과 재현이 쉬우므로, 신뢰할 만한 기존 연구물의 빠른 검토가 필요한 정책 결정권자에게도 호응이 될 수 있다. 최근 들어, 해외의 경영학 등 사회과학 분야는 믿을 만한 회색 문헌의 발굴과 비공개 문헌의 검증 방식에 관한 공유가 늘고 있으며, 국내 자연 과학계와 교육 분야의 경우 선행 연구를 영역별로 분류하는 문헌 연구를 통하여 다양한 관점에서 후행 연구를 유도하는 사례가 적지 않다. 이에, 국내 사회분야 학계도 일선 현장의 믿을 만한 정보원에서 학문적인 가치를 지닌 회색 문헌을 선별, 주제별로 지식을 체계 있게 합성 후 도식적으로 전달하는 $\mathrm{MMS}$ 연구가 시도되길 제언한다.

본 논문은 백색 문헌과 회색 문헌의 정보를 통해 선행 연구의 주요 개념, 증거의 유형과 연구 결과를 분류함으로써 학제간 연구에 활력을 주고 실용 지식의 간극을 메꿀 MMS 고찰 연구의 이해와 활용을 돕는 학문적인 함의가 있다. 특히, 전 세계의 코로나 19 와 같은 새로운 현상의 연구를 광범위하게 고찰하려는 산학계 연구자나 신흥의 고찰 방법에 관심 있을 초급 학자의 현장형 연구 발전에도 기여할 가치를 지녔다. 동 연구의 한계는 다성적 고찰의 접근과 주제범위 맵핑 연구를 참고하는 데 있어, 해외의 공학, 의학, 간호.보건 분야 외에는 $\mathrm{MMS}$ 논문이 아직 미미하여 본 저자들이 조사한 유관 문헌이 비인문사회 분야에 국한된 면이 있다. 무엇보다, 동 연구의 작업 중 $\mathrm{MMS}$ 방식을 적용한 국내 공개 문헌은 보이지 않아 다성적 고찰 논의는 국외 연구에 대거 의존한 가운데, 주제범위 고찰 연구는 SS 방식을 온전하게 적용한 간호 분야의 국내 논문을 일부 인용했다. 본 연구에 이어, 동 저자들은 교육 정보화 분야가 연구 관심이므로 최신의 온라인 교육 플랫폼 활용에 관하여 다양한 정보를 담고 있는 국내 공공 기관의 간행물 및 학위 논문을 포함한 회색 문헌을 고찰 후 기존의 학술지 논문과 합성한 $\mathrm{MMS}$ 방식의 실제 연구를 진행할 예정이다. 끝으로, 지난해 말부터 큰 용기를 내어 작업한 본 연구의 내용에 학계 동료 연구자들의 관심과 함께 건전한 비평을 바라 마지않는다.

\section{References}

[1] S. Y. Sa, H. Kim, The effects of reading post-modern picture books on the creativity and emotional intelligence of young children, The Journal of Learner-Centered Curriculum and Instruction, (2016), Vol.16, No.12, pp.1189-1206, DOI: 10.22251/jlcci.2016.16.12.1189

[2] Y. C. Kim, D. S. Lee, An inquiry on the theorizing of alternative writing in qualitative research, The Journal of Yeolin Education, (2013), Vol.21, No.1, pp.49-76, UCI: G704-001282.2013.21.1.002

[3] H. J. Seo, The scoping review approach to synthesize nursing research evidence, Korean Journal of Adult Nursing, (2020), Vol.32, No.5, pp.433-439, DOI: 10.7475/kjan.2020.32.5.433 
[4] H. J. Seo, S. Y. Kim, What is scoping review?, The Journal of Health Technology Assessment, (2018), Vol.6, No.1, pp.16-21, DOI: 10.34161/johta.2018.6.1.003

[5] C. W. Lee, Mapping: A study on maps as visualizing concept, Archives of Design Research, (2011), Vol.24, No.4, pp.363-376, UCI: G704-000241.2011.24.4.027

[6] V. Garousi, B. Küçük, Smells in software test code: A survey of knowledge in industry and academia, Journal of Systems \& Software, (2018), No.138, pp.52-81, DOI: https://doi.org/10.1016/j.jss.2017.12.013

[7] H. Arksey, L. O’Malley, Scoping studies: towards a methodological framework, International Journal of Social Research Methodology, (2005), Vol.8, No.1, pp.19-32, DOI: 10.1080/1364557032000119616

[8] F. Rowe, What literature review is not: diversity, boundaries and recommendations, European Journal of Information Systems, (2014), Vol.23, No.3, pp.241-255, DOI: 10.1057/ejis.2014.7

[9] S. K. Boell, D. Cecez-Kecmanovic, On being 'systematic' in literature reviews in IS, Journal of Information Technology, (2014), Vol.30, No.2, pp.161-173, DOI: 10.1057/jit.2014.26

[10] H. J. Kim, E. K. Kim, A systematic review of infection management education program for nursing students, The Journal of Learner-Centered Curriculum and Instruction, (2020), Vol.20, No.8, pp.1359-1375, DOI: 10.22251/jlcci.2020.20.8.1359

[11] K. S. Jung, A meta-analysis of mental health of gifted students and general students of Korea: A systematic review, The Journal of Learner-Centered Curriculum and Instruction, (2019), Vol.19, No.14, pp.983-1006, DOI: 10.22251/jlcci.2019.19.14.983

[12] S. J. Lee, Trends of research in low achievement in reading within the field of Korean education: A scoping review, Journal of Reading Research, (2020), No.57, pp.145-169, DOI: 10.17095/JRR.2020.57.6

[13] M. Petticrew, H. Roberts, Systematic reviews in the social sciences: A practical guide, Wiley-Blackwell, (2006)

[14] J. H. Shin, Postmodernism \& postmodern literature, American Studies, (1990), No.13, pp.79-137.

[15] J. H. Choo, Y. C. Kim, Postmodernism as new intellectual tradition of qualitative research: Methodological issues, The Journal of Anthropology of Education, (2012), Vol.15, No.3, pp.29-61, DOI: 10.17318/jae.2012.15.3.002

[16] V. Garousi, M. Felderer, M. V. Mäntyläd, Guidelines for including grey literature and conducting multivocal literature reviews in software engineering, Information and Software Technology, (2019), Vol.106, pp.101-121, DOI: 10.1016/j.infsof.2018.09.006

[17] J. Tyndall, How low can you go? : Toward a hierarchy of grey literature, Dream 08 Australian Library and Information Association Biennial Conference, (2008), September 2-5; Alice Springs, Australia.

[18] K. Petersen, S. Vakkalanka, L. Kuzniarz, Guidelines for conducting systematic mapping studies in software engineering: An update, Information and Software Technology, (2015), Vol.64, pp.1-18, DOI: 10.1016/j.infsof.2015.03.007

[19] P. Antil, Requirements engineering in global scaled agile software development environment: a multi-vocal literature review protocol, Technical Report SERL_TR_2018_01, Auckland University of Technology, (2018)

[20] H. Y. Cho, A Comparative Study on Usability of Grey Literature among Sciences, Using Citation Analysis, Journal of the Korean Library and Information Science Society, (2008), Vol.42, No.1, pp.273-294, UCI: G704-000226.2008.42.1.005

[21] B. A. Kitchenham, D. Budgen, O. P. Brereton, Using mapping studies as the basis for further research - A participant-observer case study, Information and Software Technology, (2011), Vol.53, No.6, pp.638-651, DOI: 10.1016/j.infsof.2010.12.011

[22] J. S. Ryu, Y, C. Kim, Collage Approach in Qualitative Research: Representation of Life History on a Korea Multicultural Elementary School Student, Journal of Qualitative Inquiry, (2015), Vol.1, No.1, pp.1-35.

[23] B. Dowdeswell, R. Sinha, S. G. MacDonell, Finding faults: A scoping study of fault diagnostics for Industrial CyberPhysical Systems, Journal of Systems and Software, (2020), Vol.168, pp.1-16, DOI: 10.1016/J.JSS.2020.110638

[24] P. Nistala, K. V. Nori, R. Reddy, Software quality models: A systematic mapping study, 2019 IEEE/ACM International Conference on Software \& System Processes, (2019), May 25; Montreal, Canada

[25] E. C, Choi, On qualitative research in Sport (Pedagogy) - A half life story of a qualitative researcher -, Journal of Qualitative 
Inquiry, (2016), Vol.2, No.2, pp.31-55.

[26] J. Adams, F. C. Hillier-Brown, H. J. Moore, A. A. Lake, V. Araujo-Soares, M. White, C. Summerbell, Searching and synthesising 'grey literature' and 'grey information' in public health: critical reflections on three case studies, Systematic reviews, (2016), Vol.5, No.1, pp.1-11, DOI: 10.1186/s13643-016-0337-y

[27] S. H. Choi, Y. C. Kim, Domain analysis as qualitative data analysis method: Exploration of various methods, Journal of Qualitative Inquiry, (2017), Vol.3, No.1, pp.133-155.

[28] J. H. Choi, Y. R. Kim, Communication of nonverbal students with severe and multiple disabilities: A systematic scoping review, Korean Journal of Physical, Multiple, \& Health Disabilities, (2020), Vol.63, No.1, pp.65-94, DOI: 10.20971/kcpmd.2020.63.1.65

[29] D. S. Oh, Y. W. Song, Introduction of an improved attorney system to spread the problem solving process in manufacturing field, Asia-pacific Journal of Convergent Research Interchange, (2021), Vol.7, No.5, pp.65-81, DOI: 10.47116/apjcri.2021.05.07

[30] J. H. Heo, Y. W. Song, TRIZ basic learning method through linkage with interests, Asia-pacific Journal of Convergent Research Interchange, (2021), Vol.7, No.5, pp.147-161, DOI: 10.47116/apjcri.2021.05.14 